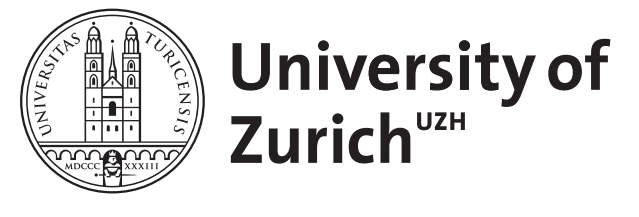

\title{
Einsatz von gecufften Endotrachealtuben bei Kindern in der Notfall- und Rettungsmedizin
}

\author{
Weiss, M ; Nicolai, T
}

\begin{abstract}
Moderne Pädiatrietuben mit Cuff kommen in der Kinderanästhesie und Kinderintensivmedizin vermehrt zur Anwendung. Hohe Treffsicherheit bei der Tubusgrößenwahl sowie die zuverlässige Abdichtung des Atemwegssystems ohne den Einsatz übergroßer Tuben sind entscheidende Vorteile gecuffter Tuben auch bei Säuglingen und kleinen Kindern in der Notfall- und Rettungsmedizin. Für einen sicheren Einsatz gecuffter Tuben in dieser Altersgruppe ist die Auswahl eines Pädiatrietubus mit korrekt platziertem Hochvolumen-Niederdruck-Cuff und Intubationstiefenmarkierung sowie eine geprüfte Tubusgrößenauswahlliste die Vorraussetzung. Die Überprüfung des Tubus auf eine Luftleckage nach der Intubation bei noch entleertem Cuff, das Füllen des Cuffs unter Cuffdruckkontrolle sowie die Cuffdruckbegrenzung auf maximal 20cmH2O erlauben den sicheren Einsatz von gecufften Tuben bei Säuglingen und Kindern. Fehlen gute Kindertuben mit Cuff, Altersgrößenauswahlliste und Cuffmanometer, so dürfen gecuffte Tuben bei Säuglingen und Kindern in der Rettungs- und Notfallmedizin nicht eingesetzt werden. Dann sollten weiterhin ungecuffte Tuben zum Einsatz gelangen
\end{abstract}

DOI: https://doi.org/10.1007/s10049-006-0805-3

Posted at the Zurich Open Repository and Archive, University of Zurich

ZORA URL: https://doi.org/10.5167/uzh-156005

Journal Article

Published Version

Originally published at:

Weiss, M; Nicolai, T (2006). Einsatz von gecufften Endotrachealtuben bei Kindern in der Notfall- und Rettungsmedizin. Notfall Rettungsmedizin, 9(2):186-189.

DOI: https://doi.org/10.1007/s10049-006-0805-3 
Notfall Rettungsmed 2006 9:186-189 DOI 10.1007/s10049-006-0805-3

Online publiziert: 14. März 2006

(c) Springer Medizin Verlag 2006

\section{Redaktion}

T. Nicolai, München

M. Weiss, Zürich

\section{Weiss ${ }^{1} \cdot$ T. Nicolai $^{2}$}

${ }^{1}$ Anästhesieabteilung, Universitäts-Kinderkliniken Zürich

${ }^{2}$ Intensivstation des Dr. von Haunerschen Kinderspitals,

Klinikum der Universität München

\section{Einsatz von gecufften Endotrachealtuben bei Kindern in der Notfall- und Rettungsmedizin}

Endotracheale Tuben mit Cuff haben in den letzten Jahren in der Kinderanästhesie wie auch in der pädiatrischen Intensivmedizin vermehrt Anwendung bei Kindern unter 8 Jahren gefunden $[1,2,3,4$, $5,6]$. Neue Kindertuben mit Cuff $[7,8,9]$ scheinen auch die Unzulänglichkeiten [10, 11, 12, 13, 14, 15, 16] bisheriger Kindertuben mit Cuff weitgehend beseitigt und damit die Sachlage verändert zu haben [17].

Die wichtigsten Vorteile und Argumente, gecuffte Tuben bereits bei kleinen Kindern einzusetzen, sind die hohe Treffsicherheit bei der Tubusgrößenwahl sowie die zuverlässige Abdichtung des Atemwegssystems ohne den Einsatz übergroßer Tuben [18]. Beide Eigenschaften machen den Einsatz von gecufften Tuben bei kleinen Kindern in der Rettungs- und Notfallmedizin bzw. in kritischen Situationen attraktiv und haben bei einigen Rettungsdiensten und Notaufnahmestationen bereits auch zu einer Umstellung auf gecuffte Kindertuben geführt.

Wenn gecuffte Tuben bei Säuglingen und Kleinkindern in der Notfall- und Rettungsmedizin eingesetzt werden, dann gilt es einige wichtige Punkte und Vorsichtsmaßnahmen zu beachten, welche im Folgenden besprochen werden sollen.

\section{Tubusgrößenwahl}

Gecuffte Tuben werden im Innendurchmesser (ID) 0,5-1,0 mm kleiner als ungecuffte Tuben gewählt, um
- den Ringknorpel möglichst wenig mit Druck durch den Tubus zu belasten,

- das Risiko, einen übergroßen Tubus einzusetzen bzw. auszuwechseln, stark zu minimieren,

- um das zusätzliche Cuffmaterial am Tubus zu kompensieren.

Heute gängige Empfehlungen zur Größenwahl von gecufften Tuben bei Kindern sind die Formeln von Khine [1] sowie von Motoyama [19] für Kinder ab 2 Jahren. Für Kinder unter 2 Jahren stützt sich die Größenwahl meist auf die Empfehlung von Khine ([1]; • Tab. 1).

Während die Innendurchmesser standardisiert sind, zeigen die Außendurchmesser von gecufften Tuben zwischen den Herstellern, aber auch innerhalb eines Herstellers [16] beträchtliche Unterschiede [10]. Da der Außendurchmesser des Tubus von entscheidender Wichtigkeit für den passenden Sitz eines Tubus im Ringknorpel ist, sind oben genannte Formeln zu Größenwahl nicht generell für alle gecufften Kindertuben anwendbar. Die Variabilität der Außendurchmesser ist im Übrigen auch für die diversen Formeln beim Einsatz ungecuffter Tuben mitverantwortlich [20]. Generell muss daher gefordert werden, dass jeder Hersteller von Kindertuben eine klinisch kontrollierte Größenwahlempfehlung für seine Tubusserien mitliefert oder gar die Tubusverpackung mit Angaben über die Alterszielgruppe bedruckt, was leider bis heute noch bei keinem Tubushersteller der Fall ist.

\section{Tab. 1 Wahl der Tubusgröße (gecuffte Tuben)}

\begin{tabular}{|llll|}
\hline \multicolumn{2}{|l}{ Tubusgrößenwahl nach Khine } & \multicolumn{2}{l}{ Tubusgrößenwahl nach Motoyoma } \\
\hline Alter [Jahre] & Innendurchmesser [mm] & Alter [Jahre] & Innendurchmesser [mm] \\
\hline $0-1$ & 3,0 & $0-1$ & 3,0 \\
\hline $1-3$ & 3,5 & $1-2$ & 3,5 \\
\hline $3-5$ & 4,0 & $2-4$ & 4,0 \\
\hline $5-7$ & 4,5 & $4-6$ & 4,5 \\
\hline $7-9$ & 5,0 & $6-8$ & 5,0 \\
\hline $9-11$ & 5,5 & $8-10$ & 5,5 \\
\hline $11-13$ & 6,0 & $10-12$ & 6,0 \\
\hline $13-15$ & 6,5 & $12-14$ & 6,5 \\
\hline $15-17$ & 7,0 & $14-16$ & 7,0 \\
\hline Tubuswechselrate (nach Khine [1]): & Tubuswechselrate (nach Dullenkopf [18]): \\
\hline $0-16$ Jahre ( $\mathrm{n}=251): 1,0 \%$ & 0-16 Jahre ( $=500): 1,6 \%$ \\
\hline Tubusmarke: Sheridan & Tubusmarke: Microcuff PET \\
\hline
\end{tabular}


Zur Treffsicherheit von Tubusgrößenformeln für gecuffte Tuben gibt es zurzeit gerade 2 klinisch-kontrollierte Untersuchungen $[1,18]$. Welche Formel man auch immer anwendet, entscheidend ist, dass ein gecuffter Tubus bei völlig entleertem Cuff eine Luftleckage bei spätestens $20 \mathrm{cmH}_{2} \mathrm{O}$ Inflationsdruck aufweisen muss. Andernfalls ist auf den nächst kleineren Tubus zu wechseln (• Tab. 1).

\section{Tubuswahl}

Aus Abbildung 1 wird deutlich ersichtlich, dass erhebliche Unterschiede in der Ausgestaltung von gecufften Kindertuben gleicher Größe (ID) von verschiedenen Herstellern vorhanden sind. Die wesentlichen Unzulänglichkeiten betreffen

- unterschiedliche Außendurchmesser bei gleichem ID,

- das Fehlen einer Intubationstiefenmarkierung,

- falsch positionierte Intubationstiefenmarkierungen (Risiko der endobronchialen Intubation),

- zu hoch am Tubusschaft positionierte Cuffs (Risiko der intralaryngealen Lage des Cuffs),

- Cuffs mit zu kleinen Residualdurchmesser, sodass der Cuff bei $20 \mathrm{~cm}$ $\mathrm{H}_{2} \mathrm{O}$ Cuffdruck das Innenlumen der Trachea nicht abzudichten vermag.

Letzteres entspricht dem Verhalten von Niedervolumen-Hochdruck-Cuffs, welche erst mit hohem Druck die Trachea abdichten im Gegensatz zu HochvolumenNiederdruck-Cuffs, welche die Trachea bereits bei niedrigen Cuffdruckwerten auskleiden und abdichten [21, 22].

Bei der Auswahl eines pädiatrischen Endotrachealtubus mit Cuff sollte darauf geachtet werden, dass der Tubus eine dünnwandige Tubuswand und einen kurzen Hochvolumen-Niederdruck-Cuff aufweist, der möglichst distal am Tubusschaft platziert ist. Polyurethan-Cuffs erlauben die Trachea bei wesentlich tieferen Cuffdruckwerten abzudichten als Polyvinylchlorid-Cuffs $[8,18,23]$.

Weiter ist es sicherlich hilfreich, wenn der Tubus eine korrekt angebrachte Tiefenmarkierung aufweist, welche dem mit pädiatrischen Formeln nicht immer vertrauten Intubierenden in der Notfallsitua-

Notfall Rettungsmed 2006 · 9:186-189 DOI 10.1007 / s10049-006-0805-3

(c) Springer Medizin Verlag 2006

\section{Weiss · T. Nicolai \\ Einsatz von gecufften Endotrachealtuben bei Kindern in der Notfall- und Rettungsmedizin}

Zusammenfassung

Moderne Pädiatrietuben mit Cuff kommen in der Kinderanästhesie und Kinderintensivmedizin vermehrt zur Anwendung. Hohe Treffsicherheit bei der Tubusgrößenwahl sowie die zuverlässige Abdichtung des Atemwegssystems ohne den Einsatz übergroßer Tuben sind entscheidende Vorteile gecuffter Tuben auch bei Säuglingen und kleinen Kindern in der Notfall- und Rettungsmedizin. Für einen sicheren Einsatz gecuffter Tuben in dieser Altersgruppe ist die Auswahl eines Pädiatrietubus mit korrekt platziertem Hochvolumen-Niederdruck-Cuff und Intubationstiefenmarkierung sowie eine geprüfte Tubusgrößenauswahlliste die Vorraussetzung. Die Überprüfung des Tubus auf eine Luftlecka- ge nach der Intubation bei noch entleertem Cuff, das Füllen des Cuffs unter Cuffdruckkontrolle sowie die Cuffdruckbegrenzung auf maximal $20 \mathrm{cmH}_{2} \mathrm{O}$ erlauben den sicheren Einsatz von gecufften Tuben bei Säuglingen und Kindern. Fehlen gute Kindertuben mit Cuff, Altersgrößenauswahlliste und Cuffmanometer, so dürfen gecuffte Tuben bei Säuglingen und Kindern in der Rettungs- und Notfallmedizin nicht eingesetzt werden. Dann sollten weiterhin ungecuffte Tuben zum Einsatz gelangen.

Schlüsselwörter

Endotracheale Intubation - Beatmungstubus Cuff $\cdot$ Kinder $\cdot$ Säuglinge

\section{Cuffed pediatric tracheal tubes in emergency medicine}

\section{Abstract}

Cuffed pediatric tracheal tubes are increasingly used in pediatric anesthesia and pediatric intensive care for infants and small children. High chance to select a correct-fitting tube and reliable sealing of the trachea without the use of an oversized uncuffed tube are the most import advantages of using cuffed tubes for infants and small children in emergency situations. If cuffed tubes in pediatric emergency are used a well-designed tube with a correctly placed high volume-low pressure and an adequate intubation depth mark and the presence of a printed chart with recommendations for age-related tube size selection should be available. Confirmation of an air leak around the trache- al tube after intubation with the cuff not inflated, cuff inflation by cuff pressure monitoring and limitation of cuff pressure at $20 \mathrm{~cm}$ $\mathrm{H}_{2} \mathrm{O}$ allows the safe use of cuffed tracheal tubes in infants and children. In case of lack of good pediatric cuffed tubes, lack of a chart with recommendations for age-related tube size selection and lack of a cuff pressure manometer, cuffed pediatric tubes should not be used in infants and children in the emergency setting.

Keywords Intubation - Tracheal tubes - Cuffed tubes . Children · Infants 


\section{Pädiatrische Notfälle}

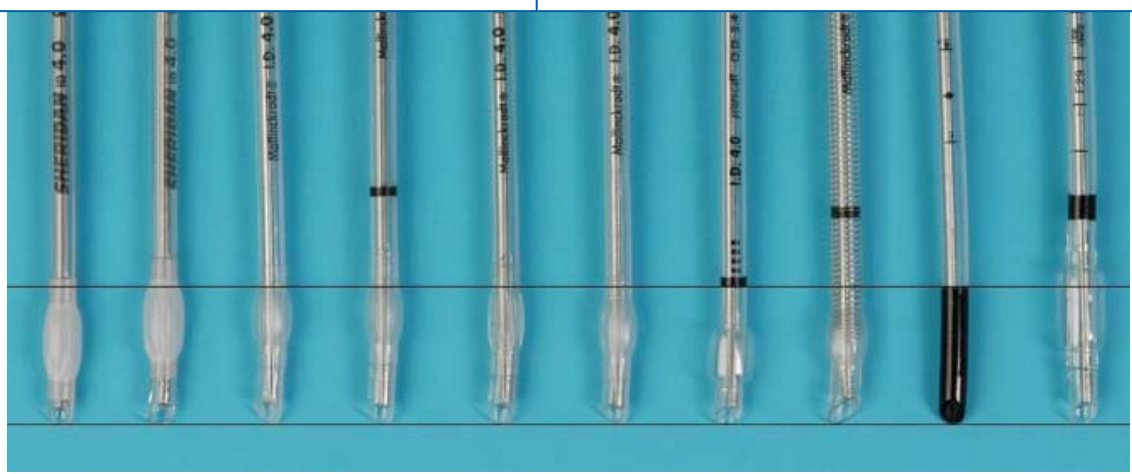

Abb. $1 \Delta$ Gecuffte Endotrachealtuben mit Innendurchmesser (ID) von 4,0 mm unterschiedlicher Hersteller sowie korrespondierender ungecuffter Endotrachealtubus der Größe ID 4,5 mm als Maßgabe für die Intubationstiefe auf Stimmbandniveau

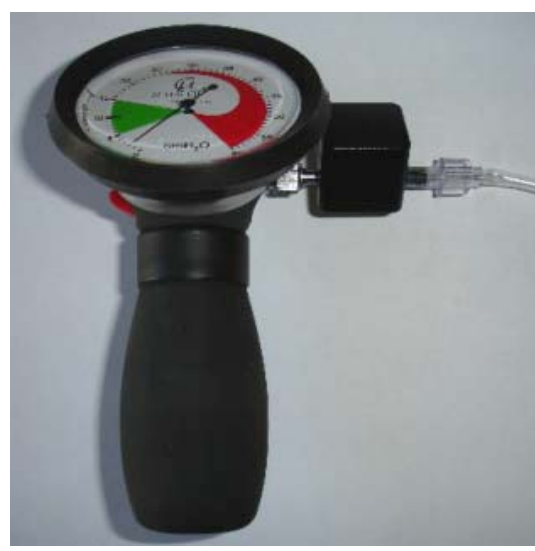

Abb. $2 \Delta$ Cuffmanometer mit Überdruckventil zur oberen Begrenzung des Cuffdrucks auf maximal $20-22 \mathrm{cmH}_{2} \mathrm{O}$ (Microcuff $\mathrm{GmbH}$, Weinheim, Germany)

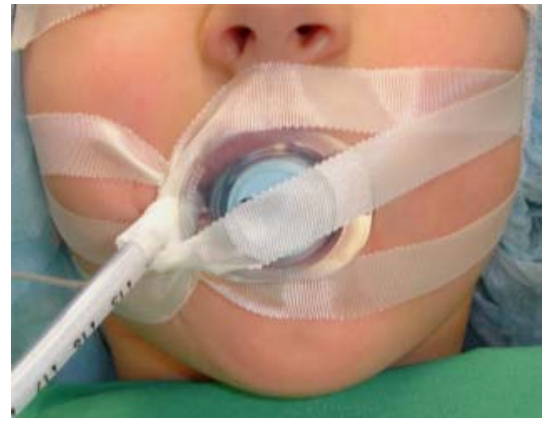

Abb. $3 \Delta$ Stabile Tubusfixation bei oral eingeführtem Tubus mit Klebestreifen über Oberund Unterkiefer sowie oropharyngealem Atemweg (Güdel) als Beißschutz und zur Tubusstabilisierung

tion hilft, den Tubus so zu platzieren, dass der Cuff sicher in der Trachea und die Tubusspitze mit einem sicheren Abstand zur Bifurkation zu liegen kommt $[24,25]$.

\section{Cuffdruck}

Die Überwachung und Begrenzung des Cuffdrucks ist entscheidend beim Einsatz von gecufften Tuben in der Pädiatrie. Hyperinflation des Cuffs führt einerseits zu Druckschädigungen der Atemwegsschleimhaut gefolgt von Ischämie, Ulzera und Narbenbildungen [26], andererseits führt das unbedachte großzügige Füllen von Cuffs bei Kindertuben zum Volutrauma der Atemwege [11, 27].

Bedingt durch das kleine Residualvolumen pädiatrischer Cuffs, führen bereits kleine Mengen an Luft aus einer Spritze oder leichte Kompression eines Cuffmanometerballons zu Cuffdruckwerten bzw. Wanddruckwerten von $>100 \mathrm{cmH}_{2} \mathrm{O}$ [28]. Erb u. Frei schlagen den Einsatz einer Insulinspritze in Kombination mit einem Cuffmanometer vor, um vorsichtig den Cuffdruck bis maximal $20 \mathrm{cmH}_{2} \mathrm{O}$ einzustellen [4]. Diese Technik sowie elektronische Cuffdruckregler mögen im Klinikbetrieb brauchbar und sinnvoll sein, für den Einsatz im Rettungsdienst sind sie jedoch eher ungeeignet.

Die Palpation des Cuffdrucks am Pilotballon zur Abschätzung des Cuffdrucks ist ebenfalls unzuverlässig [29]. Die gängige Praxis, soviel Luft in einen Tubuscuff zu füllen, bis nur noch eine ganz leichte oder knapp keine Luftleckage mehr zu hören ist, kann ebenso wenig empfohlen werden. Wie von Dullenkopf et al. gezeigt wurde, können pädiatrische Endotrachealtuben mit Cuff bis $60 \mathrm{~cm} \mathrm{H}_{2} \mathrm{O}$ Cuffdruck eine auf zu kleine Tubuscuffs, sondern auch auf eine asymmetrische Form der Trachea oder Falten- und Kanalbildung im Cuff zurückzuführen ist $[8,23]$. Luftleckage aufweisen, was nicht immer
Die Kombination eines Cuffmanometers mit einem Überdruckventil erlaubt, auch kleinvolumige Cuffs von Kindertuben sicher zu blocken, wobei der Cuffdruck bei ca. $20-22 \mathrm{cmH}_{2} \mathrm{O}$ begrenzt wird (- Abb. 2; [30]). Wird das Cuffmanometer mit Überdruckventil am Tubuscuff belassen, so wird eine akute Hyperinflation durch akzidentelle Kompression des Pilotballons oder brüske Kompression des Cuffmanometerballons ebenfalls prompt entlastet $[11,30]$.

So wie eine Cuffüberblähung vermieden werden muss, so darf ein Cuff niemals mit Unterdruck belassen werden. Cuffunterdruck führt zur Bildung von scharfen Falten, welche chronisch zu Läsionen der Atemwegsschleimhaut führen können [16]. Weist ein Tubus mit entleertem Cuff nach der Intubation eine nur knappe Luftleckage auf, so darf der Cuff niemals völlig entblockt belassen werden, sondern der Cuff muss mit wenig Luft in der Trachea unter Cuffdruckbegrenzung bis maximal $20 \mathrm{~cm} \mathrm{H}_{2} \mathrm{O}$ insuffliert werden, um allfällige Falten auszuglätten.

\section{Tubusfixation}

Der Vollständigkeit halber sei noch auf die Fixierung des Tubus hingewiesen. Die nasale Intubation bietet generell die stabilere Tubusfixation als die orale Intubation und wird daher gelegentlich von Anästhesisten bei Kindern unter 2 Jahren auch bevorzugt. Sie ist aber in der Notfallsituation nicht als primäre Intubationsroute zu empfehlen. Umso wichtiger ist es, den oral eingeführten Tubus sicher und stabil mit einem Klebestreifen über Ober- und Unterkiefer zusammen mit einem oropharyngealen Luftweg (Güdel) als Beißschutz und zur Tubusstabilisation zu fixieren (- Abb. 3). Wie bei ungecufften Tuben ist auch bei gecufften Tuben dabei zu beachten, dass von Beatmungsbeutel oder Beatmungsschläuchen kein Zug am Tubus während des Transports oder Umlagerungen ausgeübt wird.

\section{Fazit für die Praxis}

In der Kinderanästhesie und Kinderintensivmedizin werden moderne Pädiatrietuben mit Cuff vermehrt eingesetzt. Auch in der Notfall- und Rettungsmedizin be- 
Endotrachealtubus mit Cuff gemäß Tubusgrößenwahlliste auswählen

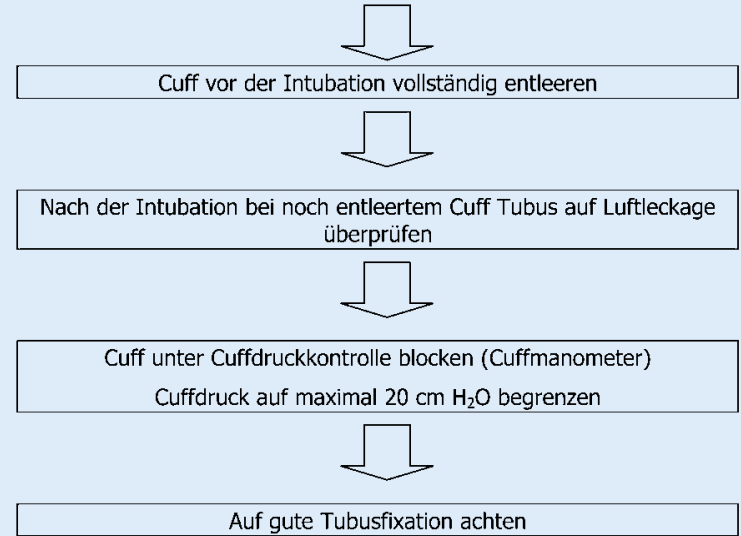

Abb. $4<$ Wichtige Punkte beim Einsatz von gecufften Tuben in der Notfall- und Rettungsmedizin stehen Vorteile beim Einsatz bei Säuglingen und kleinen Kindern. Hier sind insbesondere die hohe Treffsicherheit bei der Tubusgrößenwahl sowie die zuverlässige Abdichtung des Atemwegssystems entscheidende Vorteile. Beim Einsatz gecuffter Pädiatrietuben ist auf die Auswahl eines anatomisch korrekt gebauten Kindertubus mit distal platziertem Hochvolumen-Niederdruck-Cuff zu achten, zudem sollten eine adäquate Intubationstiefenmarkierung sowie eine validierte Tubusgrößenauswahlliste vorhanden sein. Ein Cuffmanometer muss zur Cuffdrucküberwachung bzw. -begrenzung auf maximal $20 \mathrm{cmH}_{2} \mathrm{O}$ vorhanden sein ( $\bullet$ Abb. 4). Verfügt ein Rettungswagen oder eine Notfallaufnahme nicht über gute gecuffte Pädiatrietuben mit einer Auswahlliste für die Tubusgröße und steht kein Cuffdruckmanometer zur Verfügung, so sollten gecuffte Tuben bei Säuglingen und Kindern in der Rettungsund Notfallmedizin nicht eingesetzt werden. In diesem Fall sollten weiterhin ungecuffte Tuben zum Einsatz kommen.

\section{Korrespondierender Autor PD Dr. M. Weiss}

Anästhesieabteilung, Universitäts-Kinderkliniken Steinwiesstraße 75, 8043 Zürich markus.weiss@kispi.unizh.ch
Interessenkonflikt. PD Dr. M. Weiss entwickelt in Zusammenarbeit mit Microcuff GmbH (Weinheim, Deutschland) und TYCO Health Care, Respiratory Group (Athlone, Irland) kindergerechte Beatmungstuben mit Cuff. Aus diesen Kooperationen sind weder Patentrechte, Verträge noch finanzielle Abgeltungen für PD Dr. Weiss hervorgegangen.

Trotz des möglichen Interessenkonflikts ist der Beitrag unabhängig und produktneutral.

\section{Literatur}

1. Khine HH, Corddry DH, Kettrick RG et al. (1997) Comparison of cuffed and uncuffed tracheal tubes in young children during general anesthesia. Anesthesiology 86: 627-631

2. Murat I (2001) Cuffed tubes in children: a 3-year experience in a single institution. Paediatr Anaesth 11: 748-749

3. Fine GF, Borland LM (2004) The future of the cuffed endotracheal tube. Pediatr Anesth 14: 38-42

4. Erb T, Frei FJ (2001) The use of cuffed endotracheal tubes in infants and small children. Anaesthesist 50: 395-400

5. Newth CJ, Rachmann B, Patel N, Hammer J (2004) The use of cuffed versus uncuffed endotracheal tubes in pediatric intensive care. J Pediatr 144: 333-337

6. Deakers TW, Reynold G, Stretton M, Newth C (1994) Cuffed tubes in pediatric intensive care. J Pediatr 125: 57-62

7. Weiss M, Dullenkopf A, Gerber AC (2004) Microcuff pediatric tracheal tube. A new tracheal tube with a high volume-low pressure cuff for children. Anaesthesist 53: 73-79

8. Dullenkopf A, Schmitz A, Gerber AC, Weiss M (2004) Tracheal sealing characteristics of pediatric cuffed tracheal tubes. Pediatric Anesthesia 14: 825-830

9. Frei F (2004) Kinderanästhesie, 3. Aufl. Springer, Berlin Heidelberg New York, S 140

10. Weiss M, Dullenkopf A, Gysin C, Dillier C, Gerber AC (2003) Shortcomings of paediatric cuffed endotracheal tubes. Br J Anaesth 92: 78-88

11. Holzki J (2002) Tubes with cuffs in newborn and young children are a risk! Anaesthesist 51: 321323

12. Ho AM, Aun CS, Karmakar MK (2002) The margin of safety associated with the use of cuffed paediatric tracheal tubes. Anaesthesia 57: 173-175
13. Devys JM, Schauvliege F, Talor G, Plaud B (2004) Cuff compliance of pediatric and adult cuffed tracheal tubes: an experimental study. Pediatr Anaesth $14: 676-680$

14. Goel S, Lim SL (2003) The intubation depth marker: the confusion of the black line. Paediatr Anaesth 13: 579-583

15. Wallace CJ, Bell GT (2001) Tracheal tube markings. Paediatr Anaesth 14: 279-285

16. Dillier CM, Trachsel D, Baulig W, Gysin C, Gerbe AC, Weiss M (2004) Laryngeal web due to an inap propriately designed cuffed paediatric endotracheal tube. Can J Anesth 51: 72-75

17. Schmid E, Johr M (2005) Current discussions in pediatric anesthesia. Pediatric Anesthesia Symposium in Zurich, 17.-18. September 2004. Anaesthesist 54: 163-165

18. Dullenkopf A, Gerber AC, Weiss M (2005) Tracheal sealing characteristics of a new paediatric trachea tube with high volume-low pressure polyurethane cuff. Acta Anaesthesiol Scand 49: 232-237

19. Motoyama EK (1990) Endotracheal intubation. In: Motoyama EK DP (ed) Smith's anesthesia for infants and children. Mosby, St. Louis, pp 269-275

20. Johr M (2002) The choice of tube size in children. Anaesthesist 51: 326-327

21. Guyton D, Banner MJ, Kirby RR (1991) High-volume, low-pressure cuffs. Are they always low pressure? Chest 100: 1076-1081

22. Dullenkopf A, Kretschmar O, Knirsch W et al. (2006) Comparison of tracheal tube cuff diameters with internal transverse diameters of the trachea in children. Acta Anaesthesiol Scand 50: 201-205

23. Dullenkopf A, Gerber A, Weiss M (2003) Fluid leakage past tracheal tube cuffs: evaluation of the new Microcuff endotracheal tube. Intensive Care Medicine 29: 1849-1853

24. Weiss M, Gerber AC, Dullenkopf A (2005) Appropriate placement of intubation depth marks in a new cuffed paediatric tracheal tube. Br J Anaesth 94: 80-87

25. Weiss M, Balmer C, Dullenkopf A et al. (2005) Intubation depth markings allow an improved positioning of endotracheal tubes in children. Can J Anaesth 52: 721-726

26. James I (2001) Cuffed tubes in children. Paed Anaesth 11:259-263

27. Bernet V, Dullenkopf A, Maino P, Weiss M (2005) Outer diameter and shape of paediatric tracheal tube cuffs at higher inflation pressures. Anaesthesia 60: 1123-1128

28. Morris JV, Latto IP (1985) An electropneumatic instrument for measuring and controlling the pressures in the cuffs of tracheal tubes: „the Cardiff Cuff Controller". J Med Eng Technol 9: 229-230

29. Felten ML, Schmautz E, Delaporte-Cerceau S, Orliaguet GA, Carli PA (2003) Endotracheal tube cuff pressure is unpredictable in children. Anesth Analg 97: 1612-1616

30. Dullenkopf A, Bernet V, Maino P, Weiss M (2006) Performance of a novel pressure release valve for cuff pressure control in paediatric tracheal tubes. Pediatric Anesthesia 16: 19-24 